\title{
Additional Tension Caused by Bending of a Yarn Running on a Slender Cylinder
}

\author{
By Toshiro Sasaki and Katsuaki Kuroda, Members, TMSJ \\ Technical Research Laboratory, Kurabo Industries Ltd., Neyagawa, Osaka \\ Based on the Journal of the Textile Machinery Society of Japan, Transactions, Vol, 26, No. 12, T227-232 (1973)
}

Abstract

When a yarn runs on a slender cylinder, additional tension is brought in due to the bending energy $\left(\Delta T_{1}, \Delta T_{2}\right)$ of the yarn. In the previous paper, we proposed that the additional tension is shown as $\left\{\Delta T_{1}\left(e^{\mu \theta}-1\right)+\Delta T_{2}\right\}$.

In this paper, an experiment was carried out by using a rotating slender cylinder to notice the existence of additional tension, and to get reasonable values of $\Delta T_{1}$ and $\Delta T_{2}$. However, $\Delta T_{1}$, and $\Delta T_{2}$ should be understood as components of deformation energy including bending energy.

\section{Introduction}

The frictional resistance of a yarn running on a slender cylinder is larger than the calculated value obtained by the usual capstan equation. For this reason, when a yarn runs on for example, a knitting needle, its tension becomes excessive, by high, and causes often troubles in knitting process. To solve these troubles, it is necessary to clarify this phenomenon and to estimate the value of this resistance from the yarn properties.

In our previous paper ${ }^{1)}$, we proposed the following capstan equation under the presumption that the bending energy of the yarn would be required which would be added to the yarn tension:

$$
T_{2}=T_{1} e^{\mu \theta}+\Delta T_{1}\left(e^{\mu \theta}-1\right)+\Delta T_{2}
$$

where $\Delta T_{1}$ is bending energy and $\Delta T_{2}$ is hysteresis loss in bending. Both of them are obtained from bending test of a yarn. The value $\left\{T_{1}\left(e^{\mu \theta}-1\right)+\Delta T_{2}\right\}$ is the additional tension compared with the usual capstan equation.

In this paper, we will prove this hypotesis from another view point. When a yarn running on a rotating slender cylinder, there will be no friction between the yarn and the cylinder, if there was no relative surface speed between them. Consequently, in this case the additional tension due to yarn running on a cylinder is caused only by the bending effect. In this way, we can make sure of the property of eq.(1), and can obtain some properties of $\Delta T_{1}$ and $\Delta T_{2}$.

\section{Theory}

When a slender cylinder is rotated (surface speed: $v$ ) in 30

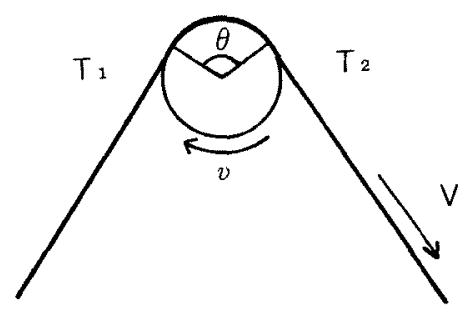

Fig. I Friction between a yarn and a rotating slender cylinder

the direction of yarn running (speed: $V$ ) as shown in Fig. 1 , the final tension $T_{2}$ can be calculated from the initial tension $T_{1}$ by the usual capstan equation, as follows:

$$
\begin{array}{lll}
\text { if } & V>v, & T_{2}=T_{1} e^{\mu \theta} \\
\text { if } & V=v, & T_{2}=T_{1} \\
\text { if } & V<v, & T_{2}=T_{1} e^{-\mu \theta} .
\end{array}
$$

The yarn tension along the yarn is shown in Fig. 2 schematically.

If the bending effect of the yarn is considered, eqs. (2), (3) and (4) are turned into eqs. (2)', (3)' and (4)' as follows:

$$
\begin{array}{lll}
\text { if } \quad V>v, & T_{2}=T_{1} e^{\mu \theta}+\Delta T_{1}\left(e^{\mu \theta}-1\right)+\Delta T_{2} \\
\text { if } \quad V=v, & T_{2}=T_{1}+\Delta T_{2} \\
\text { if } \quad V<v, & T_{2}=T_{1} e^{-\mu_{\theta}}+\Delta T_{1}\left(e^{-\mu_{\theta}}-1\right)+\Delta T_{2}
\end{array}
$$

Equation (2)' is identical with eq. (1). Tension derived from eqs. $(2)^{\prime},(3)^{\prime}$ and $(4)^{\prime}$ is shown in Fig. 3, respectively.

These equations are derived from the following considerations. The bending energy will be required when a yarn comes contact with a slender cylinder, and the unbending energy $\left(-\Delta T_{1}+\Delta T_{2}\right)$ will be required when the yarn

$$
\text { Journal of The Textile Machinery Society of Japan }
$$




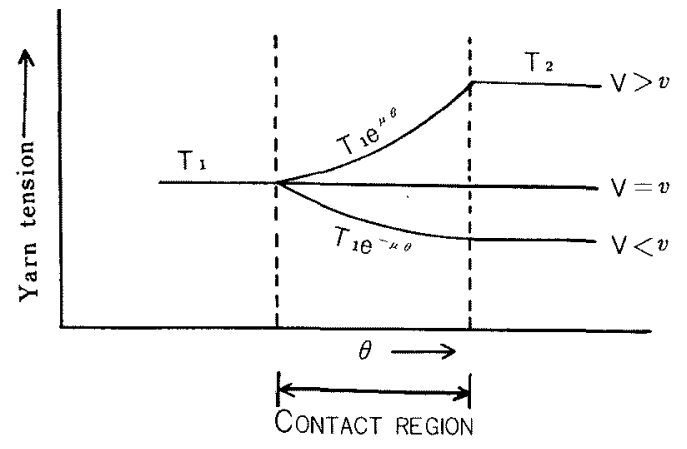

Direction of yarn $\longrightarrow$

Fig. 2 Variation of yarn tension (1)

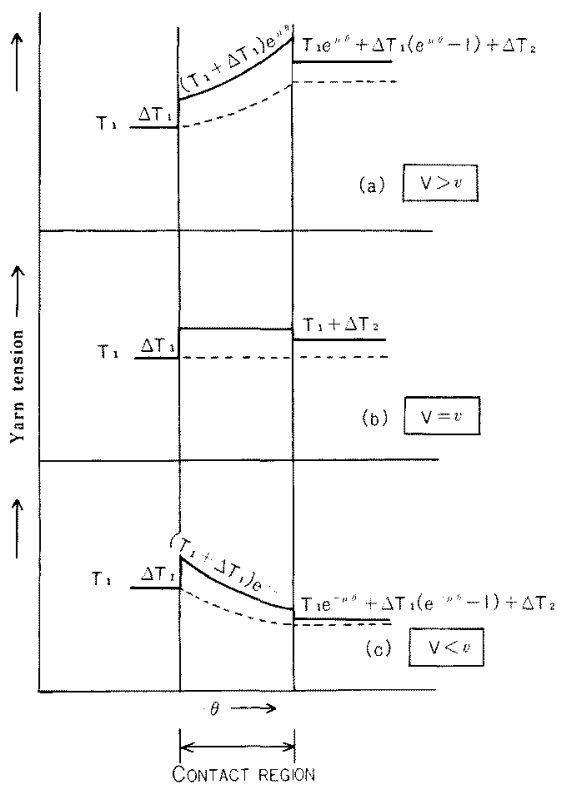

Direction of yarn advance $\longrightarrow$

Fig. 3 Variation of yarn tension (2)

detaches from the cylinder, and both energy causes additional yarn tention on each position.

Hereupon, let us consider the change of $T_{2}$ when $V$ is increased gradually with $v$ fixed. Since the direction of the frictional force at $V>v$ is opposite to that at $V<v$, the change of $T_{2}$ against $V$ will become discontinuous as shown in Fig. 4. As the friction between the yarn and the cylinder does not occur at $V=v$, the tension additional to $T_{1}$ is only due to bending hysteresis loss $\Delta T_{2}$ as shown in eq. (3)' and Fig. 4(b). Therefore, $\Delta T_{2}$ could be obtained immediately from this experiment.

Vol. 20 No. $1(1974)$

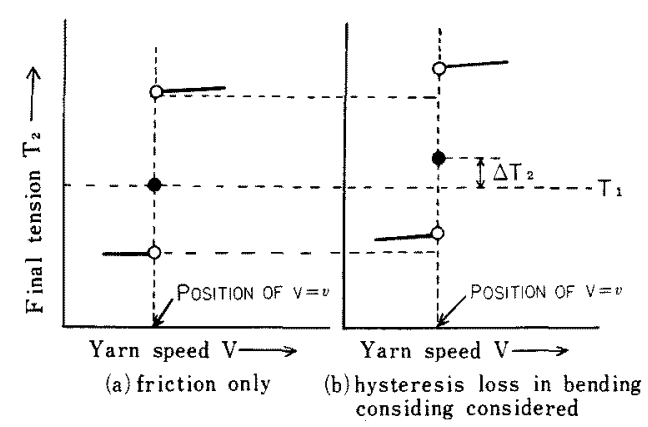

Fig. 4 Variation of final tension against yarn speed. Surface speed of the cylinder was kept constant

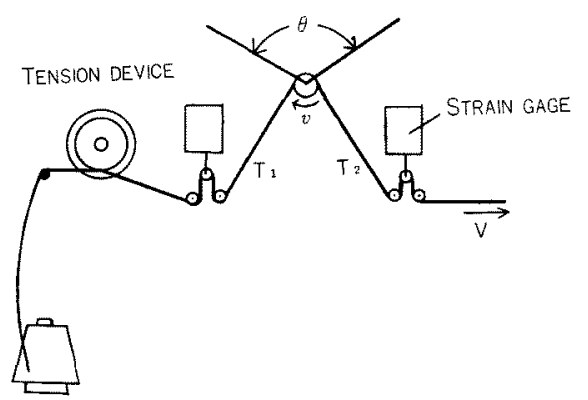

Fig. 5 Experimenal mathod

\section{Experimental}

In Fig. 5, the rotation of the cylinder was kept constant at 2,400 rpm and the yarn tension, $T_{1}$ and $T_{2}$, was measured by increasing the yarn speed. Eight cylinders, 0.26 $2.0 \mathrm{~mm}$ in diameter, were used. Initial tension was kept at $20.5 \mathrm{~g}$, and the contact angle $\theta$ was $130^{\circ}$.

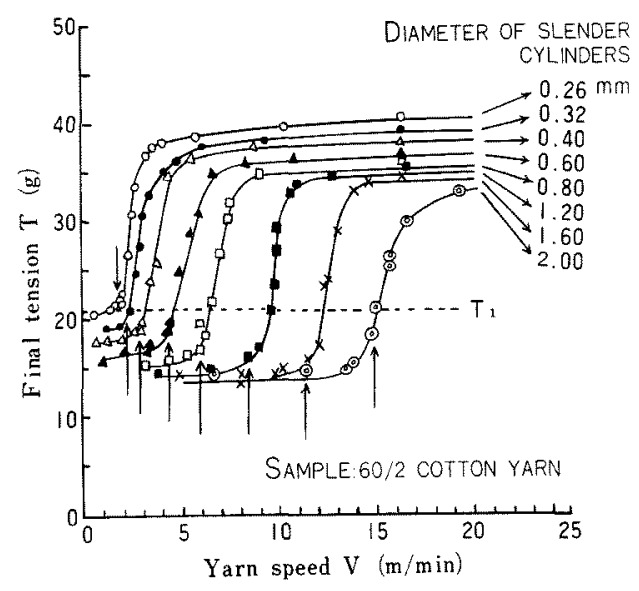

Fig. 6 Relation between final tension and yarn speed 


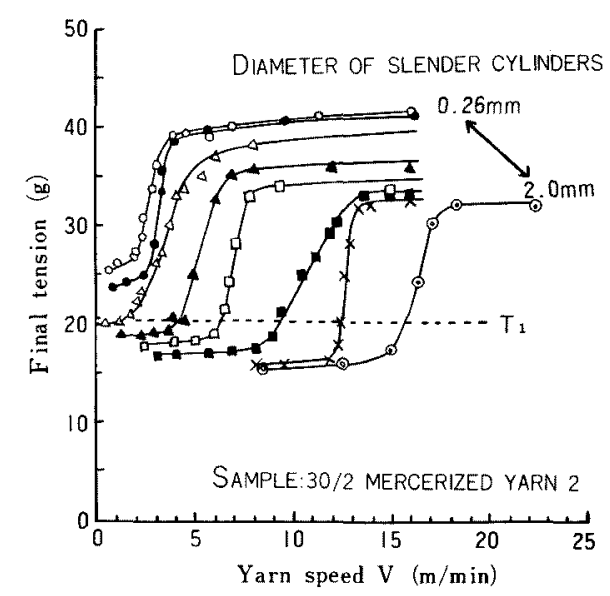

Fig. 7 Relation between final tension and yarn speed

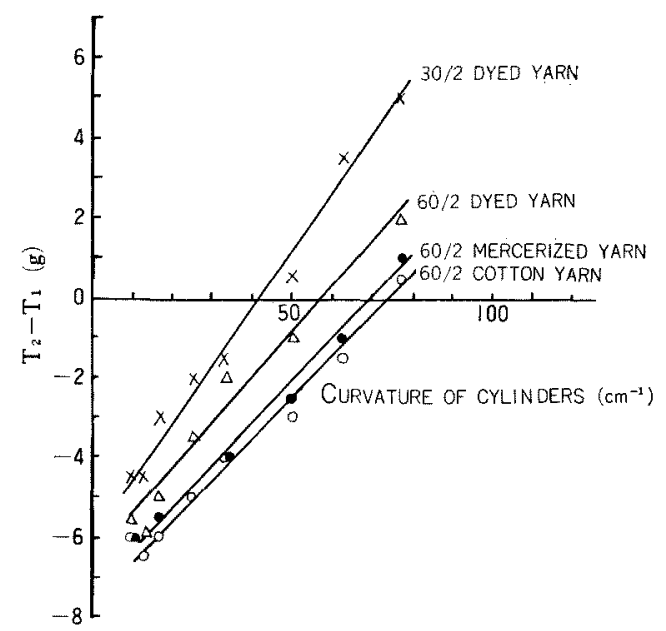

Fig. 8 Relation between $\left(T_{2}-T_{1}\right)$ and curvature of slender cylinders when $V<v$

\section{Results and Discussions}

Figure 6 shows the tension $T_{2}$ versus yarn speed for $60 / 2$ cotton yarns. Symbol $\uparrow$ in the figure shows the position of $V=v . T_{2}$ varies steeply near $V=v$, but discontinuity does not appear contrary to our expection. Besides, $T_{2}$ increases as the cylinder diameter becomes small.

Figure 7 is the result for dyed $30 / 2$ cotton yarns. $T_{2}$ is larger on the whole than that for $60 / 2$ yarn. Hereupon, we pay attension to $T_{2}$ when $V<v . T_{2}$ ought to be smaller than $T_{1}$ when only frictional effect acts upon the yarn. But it is evident that $T_{2}$ is larger than $T_{1}$ on cylinders having diameters of 0.26 and $0.32 \mathrm{~mm}$. The value of $\left(T_{2}-T_{1}\right)$ at $V<v$ was plotted against the curvature for 4 samples in Fig. 8. For each samples, $\left(T_{2}-T_{1}\right)$ becomes positive when the curvature is large. This means that there is tension not only by frictional effect but also by bending effect.

\section{Calculation of $\Delta T_{1}$ and $\Delta T_{2}$}

Discontinuity of $T_{2}$ was not obtained from Figs. 6 and 7. So, we could not obtain $\Delta T_{2}$ immediately from the experiment described in Section 2. Then we would consider another means.

$T_{2}$ can be measured accurately in both regions of $V>v$ and $V<v$, because $T_{2}$ is almost independent of the yarn speed except the steep slope near $V=v$. Besides, such a state as $V=v$ in which the yarn friction does not occur ought to exist in the steep slope region.

The final tension at $V>v$, is put as $T_{2}$, that at $V=v$ as $T_{2}{ }^{\prime \prime}$, and that at $V<v$ as $T_{2}{ }^{\prime}$, respectively. Then, eq. (5) is obtained from eqs. $(2)^{\prime}-(4)^{\prime}$.

$$
T_{2}^{\prime \prime}=\frac{T_{2}+T_{2} e^{\mu \theta}}{e^{\mu \theta}+1}
$$

If the frictional coefficient $\mu$ is measured by other experiments, it is able to calculate the value of $T_{2}^{\prime \prime}$ from eq. (5).

Measured $\mu$ for $60 / 2$ mercerized cotton yarns is shown in Fig. 9. It decreases with the increase of curvature.

Figure 10 shows the change of $T_{2}$ against $V$ for the same sample as used in Fig. 9. Solid circles in the figure are $T_{2}{ }^{\prime \prime}$ calculated by eq. (5) using $\mu$ of Fig. 9. From eq. (3), it is evident that the difference between this $T_{2}{ }^{\prime \prime}$ and the initial tension $T_{1}$ means the bending hysteresis loss $\Delta T_{2}$ of the yarn.

The value of $\Delta T_{2}$ obtained on the assumption that eqs. $(2)^{\prime}-(4)^{\prime}$ should hold seems reasonable, because it is very small when the cylinder is $2.0 \mathrm{~mm}$ in diameter. Besides, $\Delta T_{1}$ obtained by substituting $\Delta T_{2}$ into eq. (1) had the similar tendency to $\Delta T_{2}$.

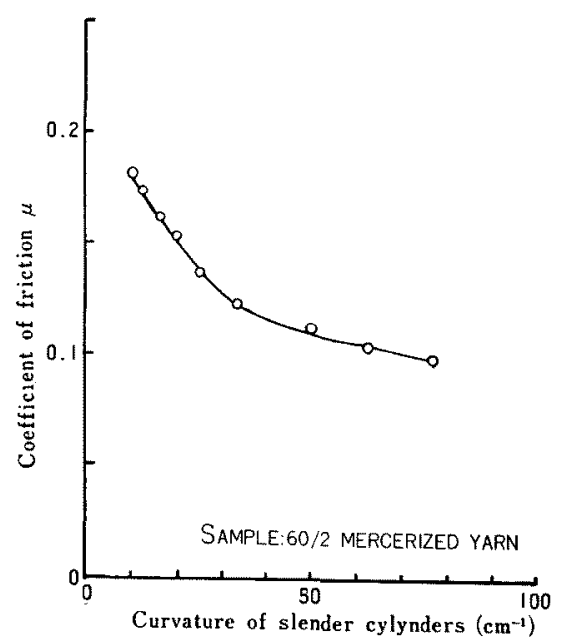

Fig. 9 Coefficent of friction $\mu$ of sample yarns

Journal of The Textile Machinery Society of Japan 


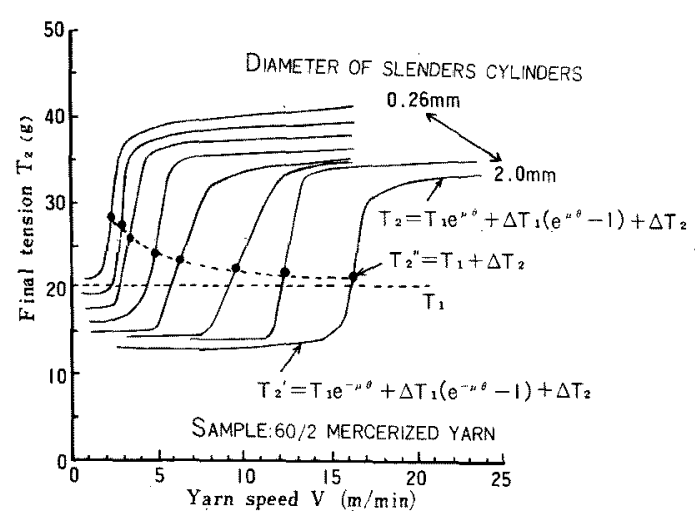

Fig. 10 Calculated final tension at $V=v$

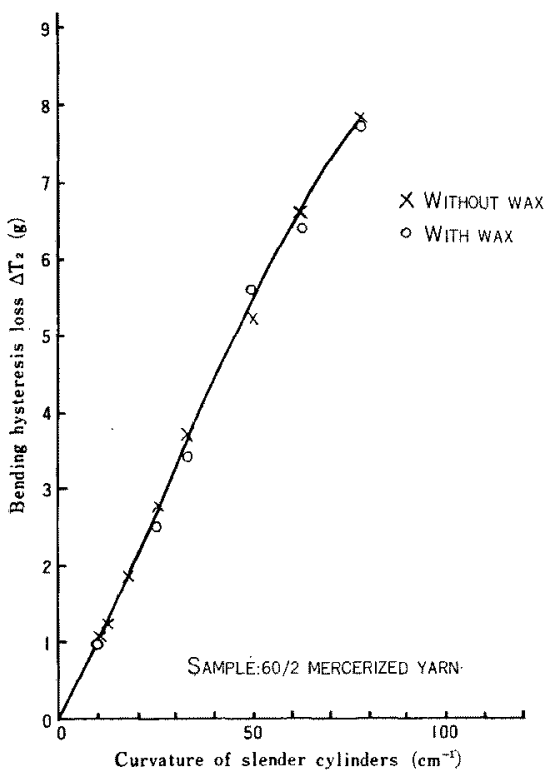

Fig. $11 \Delta T_{2}$ of yarns with wax and without wax

Next, the same $60 / 2$ mercerized yarn was passed through wax treatment, and was examined by the same method. It is generally natural that the slippability of a yarn is changed by wax treatment but bending properties do not change. So, the value of $\Delta T_{2}$ of a waxed yarn ought to be the same as that of the original yarn. Figure. 11 shows this clearly, although the values of $T_{2}$ of both yarns were different.

$\Delta T_{2}$ obtained by this method, however, is larger than that estimated from bending hysteresis measurement of the yarn. At first, we defined $\Delta T_{1}$ and $\Delta T_{2}$ as the bending energy and the hysteresis loss of a yarn. But, it seems appropriate to consider that $J T_{1}$ and $\Delta T_{2}$ are synthetic deformation energy containing bending energy. Deformation energy is required when a yarn contacts with a slender cylinder, and recovery energy from deformation $\left(-\Delta T_{1}+\Delta T_{2}\right)$ is required when the yarn detaches from the cylinder, and both enrgy changes into yarn tension on each position respectively.

In our previous paper ${ }^{1)}$, we described that other effects were expected in addition to bending energy, because the difference of final tension, between calculated by eq. (1) and measured, increases with increasing of initial tension. But, the view point mentioned above will solve these problems.

It is the subject for further study to make clear the properties of $\Delta T_{1}$ and $\Delta T_{2}$ and to explore the method to mesure them directly.

\section{Conclusion}

Additional tension caused by bending energy of a yarn was studied by using a rotating slender cylinder. Obtained results were as follows:

(1) Bending energy $\left(\Delta T_{1}, \Delta T_{2}\right)$ of a yarn was calculated by a previously derived capstan equation, $T_{2}=T_{1} e^{\mu \theta}+\Delta T_{1}\left(e^{\mu s}-1\right)+\Delta T_{2}$. Bending test of the yarn showed that the equation holds good.

(2) It is appropriate that $\Delta T_{1}$ and $\Delta T_{2}$ mean synthetic deformation energy including bending energy.

\section{Literature Cited}

[1] T. Sasaki et al; Preprints presented at Annual Convention of Text. Mach. Soc. Japan held June, 1973. (p. 75). 